\title{
Why do we offer palliative care so late to patients with liver disease?
}

Patients with advanced liver disease carry a high symptom burden and short life expectancy, but receive limited supportive and palliative care ${ }^{1}$. Providing planned day care for large volume paracentesis reduced costs, inpatient bed days and probability of dying in hospital after an unplanned admission ${ }^{1}$. These recent findings illustrate how care to people with advanced liver disease can be improved with the burden on acute service alleviated by the adoption of day-case models across the UK. This point is timely as palliative care provision for this group is poor, often occurring near to the patients' death ${ }^{2}$. Why is this? It is recognised that prognosis or future care needs are often not discussed with liver patients until the very late stages of the disease. Clinicians report that they lack the expertise and confidence to initiate these discussions and a limited understanding about what palliative care can offer, and believe that patients have negative expectations of palliative care referral ${ }^{2}$. Conversations regarding palliation often introduces family members into the patient and clinician relationship, and family members may be resistant to the idea of palliative care ${ }^{2}$.

Furthermore, many clinicians find it difficult to identify the point of 'deterioration' due to the fluctuating disease trajectory of advanced cirrhosis.

Liver clinicians perceive themselves as poor prognosticators, but patients in the last year of life share common clinical characteristics warranting referral to palliative care, such as frequent hospital admission and heavy symptom burden ${ }^{2}$. Prognostic tools now exist which help to identify when referral to specialist palliative care may be beneficial ${ }^{3}$. Perceived barriers about the appropriateness of palliative care in patients with advanced liver disease can be broken down if liver and palliative care clinicians work jointly at an early phase ${ }^{4}$. Increasingly, there is collaborative working in joint clinics and in developing shared care pathways to facilitate patient-centred and cost-effective care. Although these interventions are at a developmental stage, initial results show their potential in improving patient outcomes and promoting open end of life discussions ${ }^{5}$. They may be instrumental in mutually educating liver clinicians as to what palliative care can offer, and palliative care clinicians in aspects of advanced liver disease management ${ }^{2}$. Further work is needed to address the cost-effectiveness of these interventions, in addition to supporting clinicians in their communication with patients, improving understanding of patients' care needs and improving patients' understanding of their disease.

Authors: Joseph Low ${ }^{1}$, Aileen Marshall2, Catherine Carroll2 ${ }^{2}$, Rachel Craig ${ }^{3}$, Sarah Davis ${ }^{1}$, Anna Gola ${ }^{1}$, Lynda Greenslade ${ }^{2}$, Nicola Henawi ${ }^{3}$, Paddy Stone ${ }^{1}$, Victoria Vickerstaff ${ }^{1}$, Jo Wilson ${ }^{3}$, Douglas Thorburn².

References:

1. Hudson B, Round J, Georgeson B et al. Cirrhosis with ascites in the last year of life: a nationwide analysis of factors shaping costs, health-care use and place of death in England. Lancet Gastroenterol Hepatol 2017

2. Low J, Vickerstaff $S$, Davis $S$ et al. Advanced chronic disease in the last year of life: a mixed methods study to understand how care in a specialist liver unit could be improved. BMJ Open 2017; 7 e016887. Doi: 10.1136/bmjopen-2017-016887.

3. Highet G, Crawford D, Murray SA, et al. BMJ Supportive \& Palliative Care 2013; 4. doi:10.1136/bmjspcare2013-000488

4. Low J, Vickerstaff V, Davis $S$ et al. Palliative care for cirrhosis: a UK survey of health professionals' perceptions perceptions, current practice and future needs. Frontline Gastroenterology 2016; 7: 4-9.

5. Campbell V, Quinn S, Sikka K et al. Sooner rather than later. Early hospice intervention in advanced liver disease (ALD). British Association for Liver conference 2017.

\section{Affilitations:}

${ }^{1}$ Marie Curie Palliative Care Research Department, Division of Psychiatry, University College London, $6^{\text {th }}$ Floor, Maple House, 149 Tottenham Court Road, London W1T 7NF. 
2Sheila Sherlock Liver Unit, Royal Free Hospital \& University College London Institute of Liver and Digestive Health, UCL Royal Free Campus, Pond Street, London, NW3 2QG.

${ }^{3}$ Department of Palliative Care, Royal Free London NHS Foundation Trust, Pond Street, London, NW3 2PF.

Names and full addresses of author/co-authors' institution

Dr Joseph Low, Marie Curie Palliative Care Research Department, Division of Psychiatry, University College London, $6^{\text {th }}$ Floor, Maple House, 149 Tottenham Court Road, London W1T 7NF. Telephone: 02076799718 Fax: 0207679 9426. Email: joseph.low@ucl.ac.uk 